\title{
Electrostatic Transducer for Ultrasound Ranging Based on In-Plane Electrode Motion
}

\author{
Jorge M. Monsalve ${ }^{1}$, Franziska Wall ${ }^{1}$, Marcel Jongmanns ${ }^{1}$, Sergiu Langa ${ }^{1}$, Marco Kircher ${ }^{1}$, Bert Kai- \\ ser ${ }^{1}$, Hermann A.G. Schenk ${ }^{2}$ \\ 1 Fraunhofer Institute for Photonic Microsystems, Maria-Reiche-Str. 2, 01109 Dresden, Germany, \\ ${ }^{2}$ Arioso Systems GmbH, Maria-Reiche-Str. 1, 01109 Dresden, Germany \\ jorge.mario.monsalve.guaracao@ipms.fraunhofer.de
}

\begin{abstract}
Summary:
A Micromachined Ultrasound Transducer (MUT) based on in-plane actuation is presented in this paper. Its building block, unlike membrane-based MUTs, is a Coulomb-actuated microbeam that is deflected in the in-plane direction. This system is designed to operate at low-frequency ultrasound waves, aiming for gesture recognition applications. The sensing function is demonstrated in this work, complementing an earlier report on its transmission characteristics.
\end{abstract}

Keywords: ultrasound detection, electrostatic transducers, gesture recognition, Duffing oscillator, capacitive measurement

\section{Introduction}

Ultrasound ranging has been proven an effective means to detect hand gestures and thus control electronic devices without the need of physical contact with a screen [1]. This detection strategy is based on the periodical emission of ultrasound pulses that are reflected by the motion of the hand and detected by an array of sensors at different positions, obtaining a measurement of the time of flight of the pulse [2]. The building unit of such a device is a Micromachined Ultrasound Transducer (MUT) designed to oscillate at frequencies in the low ultrasound range (near $40 \mathrm{kHz}$ ), so that absorption losses are diminished [2]. The two main types of MUTs (piezoelectric [1-4] and capacitive [5-7]) have been implemented for the generation (and detection) of low-frequency ultrasound waves; however, we argue that these two categories belong to the family of out-ofplane transducers, in contrast to in-plane ones. Whereas out-of-plane transducers excite a membrane in the direction of the propagation of sound, in-plane transducers oscillate perpendicular to this direction and can therefore use the transverse area (depth) of the device to displace a volume of air, instead of its surfacewhich heavily determines the cost of a chip. Inplane transduction has already been implemented in the acoustic range, both for a capacitive all-silicon loudspeaker [8] and a piezoresistive microphone [9]. Here we present an extension of this principle to the ultrasound range with a capacitive transducer based on clamped- clamped microbeams tuned to operate at 40 $\mathrm{kHz}$ and requiring a low bias voltage (24V).

\section{Description of the System}

The building block of this transducer is a classical Coulomb-actuated microbeam [10], i.e. a prismatic beam of dimensions in the $\mu \mathrm{m}$-range that is moved towards a fixed electrode by means of a Coulomb force (See Fig. 1). As the beam is deflected, it displaces a volume of air towards a slit. Each microbeam behaves, therefore, as a moving wall between two acoustic chambers. A certain leakage across the beam is expected to occur, but the narrow clearance $(1 \mu \mathrm{m})$ makes this flow negligible. The microbeams are fabricated by deep reactive ion etching on a silicon-on-insulator substrate, using a similar procedure as reported in [8] for the nano e-drive microspeaker-we thus codename this transducer as NED-MUT.

A closely packed array of 258 of these beams actuated with the same voltage comprises one transmitter unit. For the receiving units, two design options were explored: one with clamped-clamped beams (152 in total), and one with clamped-free beams (410 in total). The resonance frequency of the transmitter beams lies at $53 \mathrm{kHz}$ to ensure stability when oscillating near $40 \mathrm{kHz}$, around which the resonance of the receivers was tuned. The whole device consists of 9 units of $3 \times 3 \mathrm{~mm}^{2}: 4$ receiver units on the corners of the chip and 5 transmitter units elsewhere. The electrostatic gap, which is limited by the DRIE etching process, was set to $2.5 \mu \mathrm{m}$. The arrangement of the transmitter 
units allows modifying the irradiation pattern by introducing phase shifts.

(a)

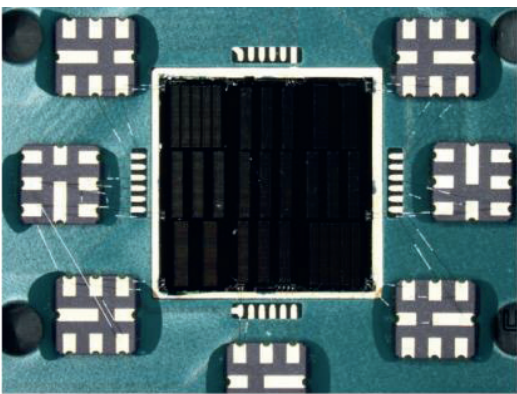

Slit at the front side

(b)

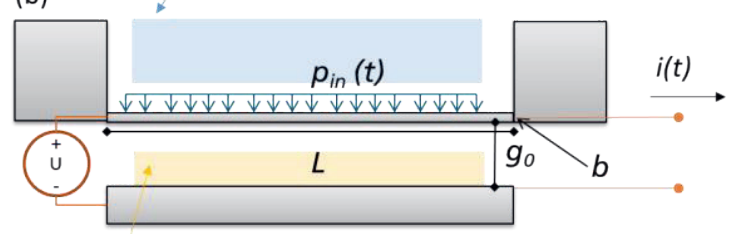

Slit at the back side

Fig. 1. (a) Photograph of the electrostatic NEDMUT and (b) scheme of one of its clamped-clamped beams acting as ultrasound receiver.

We already reported a first characterization of the transmitter function, reaching a pressure ${ }^{1}$ of $87 \mathrm{~dB}$ at a distance of $8.9 \mathrm{~cm}$ with an excitation of $24 \mathrm{~V} \mathrm{DC}$ and $24 \mathrm{~V} \mathrm{AC} \mathrm{[11]-very} \mathrm{similar} \mathrm{to} \mathrm{the}$ pressure reported in [5] but using a much lower bias voltage. Here we further report on the function of the sensing units, therefore showing that this device is capable of performing pulse-echo tests.

\section{Results}

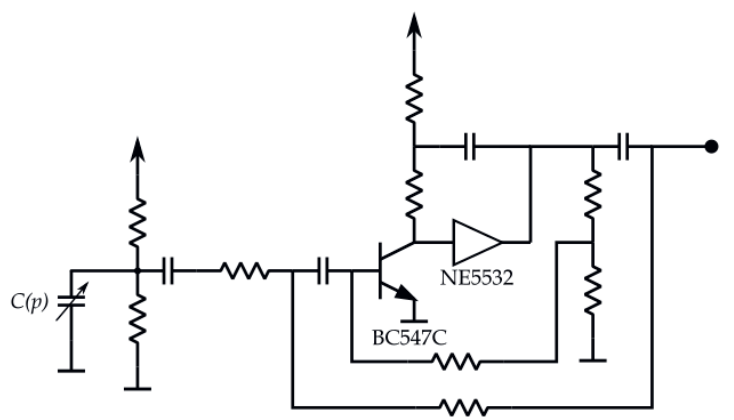

Fig. 2. Illustration of the amplifier topology used to measure the output of the in-plane ultrasound transducer.

In order to test the sensing function of the NEDMUT, a ceramic transmitter (UST-40T) was set $10 \mathrm{~cm}$ apart from the chip, hence generating a pressure of $60 \mathrm{~Pa}$ rms at $40 \mathrm{kHz}$ (see datasheet [12]). The sensing unit was connected to the amplifier shown in Fig. 2, which relies on the $h_{f e}$ factor of the transistor to amplify the signal. An output signal of $4.5 \mathrm{~V}$ peak-to-peak was meas- ured with this procedure. When no pressure is excited, a noise level of $0,5 \mathrm{Vpp}$ is measured at $38 \mathrm{kHz}$, and if a reflective object is placed inbetween, the amplitude of the $40 \mathrm{kHz}$ signal is nearly halved. The NED-MUT is thus capable of acting a as ultrasound receiver.

\section{References}

[1] D. Horsley, Beyond Touch: Tomorrow's Devices Will Use MEMS Ultrasound to Hear Your Gestures, IEEE Spectrum, (2016) Available: https://spectrum.ieee.org/semiconductors/devices /beyond-touch-tomorrows-devices-will-usemems-ultrasound-to-hear-your-gestures.

[2] D. M. van Willigen, E. Mostert and M. A.P. Pertijs, In-Air Ultrasonic Gesture Sensing with MEMS Microphones, SENSORS 2014 IEEE (2014)

[3] P. Gijsenberg, A. Halbach, Y. Jeong et al., Characterization of polymer-based piezoelectric micromachined ultrasound transducers for shortrange gesture recognition applications, J. Micromech. Microeng., vol. 29 (2019); doi: 10.1088/1361-6439/ab1f41

[4] M. Saad, C. J. Bleakley, V. Nigram, P. Kettle, UItrasonic hand gesture recognition for mobile devices, J. Multimodal User Interfaces, vol. 12, pp 31-39, (2016) DOI: 10.1007/s12193-017-0257-8

[5] I. O. Wygant, M. Kupnik, J. C. Windsor et al., 50 kHz Capacitive Micromachined Ultrasonic Transducers for Generation of Highly Directional Sound with Parametric Arrays, IEEE T. Ultrason. Ferr., vol. 56, no. 1, (2009).

[6] A. Unger, Air coupled ultrasonic transducers for industrial applications, Ph. D. dissertation, Dept. Elec. Eng., T. U. Darmstadt, Darmstadt, Germany, (2019)

[7] H. Zhang, D. Liang, Z. Wang, et al, Fabrication and Characterization of a Wideband LowFrequency CMUT Array for Air-Coupled Imaging IEEE Sensors Journal vol. 20, no. 23 (2020); doi: 10.1109/JSEN.2020.3007068

[8] B. Kaiser, S. Langa, L. Ehrig et al., Concept and proof for an all-silicon MEMS micro speaker utilizing air chambers, Microsystems \& Nanoengineering, vol. 5, no. 43 (2019).

[9] J. Czarny, Conception, fabrication and characterization of a MEMS microphone, Ph. D. dissertation, Lyon INSA, France (2015).

[10] M. I. Younis and A. H. Nayfeh, A study of the nonlinear response of a resonant microbeam to an electric actuation, Nonlinear Dynamics, vol. 31, pp. 91-117, (2003).

[11] J. M. Monsalve, M. Kircher, F. Wall et al, First Time of nanoscopic electrostatic drives pushing for ultrasonic transmission for gesture recognition, 2020 IEEE International Ultrasonics Symposium (IUS) (2020); doi: 10.1109/IUS46767.2020.9251316

[12] 400RT/R160 Air Ultrasonic Ceramic Transducers

${ }^{1}$ relative to $20 \mu \mathrm{Pa}$ rms 